\title{
The Constructionist Learning Approach in the Digital Age
}

\author{
Ilya Levin, Dina Tsybulsky \\ School of Education, Tel Aviv University, Tel Aviv, Israel \\ Email: ilia1@tauex.tau.ac.il
}

How to cite this paper: Levin, I., \& Tsybulsky, D. (2017). The Constructionist Learning Approach in the Digital Age. Creative Education, 8, 2463-2475. https://doi.org/10.4236/ce.2017.815169

Received: November 24, 2017

Accepted: December 19, 2017

Published: December 22, 2017

Copyright $\odot 2017$ by authors and Scientific Research Publishing Inc. This work is licensed under the Creative Commons Attribution International License (CC BY 4.0).

http://creativecommons.org/licenses/by/4.0/

\begin{abstract}
The article deals with the evolution of the constructionist learning approach from the beginning of the era of information technologies through the digital age. The evolution of constructionism is demonstrated in connection with two associated processes: changes in the human worldview related to the digital shift, and the corresponding transformations in human society. The study examines the evolution of basic constructionist ideas: 1) "microworlds" as "incubators of knowledge"; 2) a child as "the architect of his [or her] intelligent structures"; 3) the computer as "a machine that brings back a natural character to learning"; 4) coding as a "universal learning activity" that enables the study of fundamental scientific ideas. The constructionist ideas are analyzed in the context of today's digital reality. The main contribution of the study is formulating the changes in classical constructionism as transformations that correspond to worldview components: activating the perception of self; democratization of the mutual interactions with others; virtualization of the conception of reality; integration the subject and object in their interaction with reality.
\end{abstract}

\section{Keywords}

Digital Age, Learning, Constructionism, Information Technology, Worldview

\section{Introduction}

About forty years ago, with the advent of new information technologies (IT), marked by the creation of the personal computer (PC), Seymour Papert (1928-2016) suggested his interpretation of IT and the correspondingly new pedagogical perspectives (Papert, 1980). He was not a researcher in the field of cultural studies, sociology, psychology, or education. Papert was a mathematician. Therefore, his very interest in the personal computer is noteworthy. Papert 
realized before others that the PC is not just a new computing device, but that it constitutes an entirely novel object that could be harnessed as a universal learning tool. This fresh and unexpected view was the starting point of his remarkable ideas in the field of education.

The end of the 1970s was a time when technology was not perceived as something closely related to human life. After all, IT was then still unknown, and it was the PC that was the "first sign" of our current way of life, when the ubiquitous and unlimited access to any sort of information has become a matter of fact for the many people equipped with various mobile devices. Today, it is extremely difficult to imagine our life without digital technologies, and, for the new generation-it is even impossible. Although completely forgotten and now barely believable, in those times technology was a domain inaccessible to non-professionals, ITs were at an embryonic level and, most importantly, people were said to be separated from computing resources by the backs of programmers. Nowadays, to consider technology as something separable or far from one's reach is not only difficult, but quite inconceivable. Although the majority of experts at the time perceived the PC as something new with the potential to radically change our lives, they nevertheless treated it as an important innovation, but only in the field of technology.

Papert, by contrast, viewed the PC as the precursor of the upcoming IT epoch, which eventually would alter human consciousness entirely. Sigmund Freud (1856-1939) considered a transformation in human consciousness (i.e., in people's worldviews) as a symptom of any major revolution. He mentioned three global revolutions in the history of mankind (Freud, 1955). The first, the Copernican revolution, parted with the concept of mankind being at the center of the Universe. The second, the Darwinian revolution, led to the understanding that the human being is not a unique creation, but rather comprises part of nature, and in fact is a product of another animals' evolution. The third revolution, the Freudian one, repealed the conviction that human consciousness is fully predictable. These three revolutions have one common feature: they are not typical revolutions, in the sense that they are of not of a technological, social, or political nature. They are revolutions in human consciousness. We live in the era of the fourth revolution in the history of mankind-the digital revolution, which is supplanting our notion of a person as an isolated Cartesian object with the idea of a person as an information organism (Floridi, 2014).

Looking back, it becomes clear that in recognizing the PC's potential as a new cognitive means, Seymour Papert, actually declared the IT revolution to be a revolution in human consciousness.

A number of studies have provided a retrospective analysis of the constructionist ideas of Seymour Papert and analyzed his heritage from a current point of view (Beynon, 2016; Blikstein, 2013; Feurzeig, 2010; Resnick, 2012; Rieber, 2004). For example, a paper with the symbolic title "Gears of Our Childhood..." (Blikstein, 2013) surveys the evolution of Papert's "object-to-think-with" ideas, from the programmable bricks, through the establishment of the construction- 
ism in education movement, to the emergence of the "smart" environment. The author defines four generations of educational robots, an evolution that he views as resulting from the coexistence of two main forces: the technological and the epistemological. He demonstrates that the design practices of today's children are increasingly oriented to the research developments of the early 1980s. In other words, despite the unprecedented success of the technological force, the main challenge is epistemological: to provide for all children opportunity to work with devices as with the gears of their childhood.

The paper of Beynon (2016), titled "Mindstorms Revisited: Making New Construals of Seymour Papert's Legacy" is rooted in a famous declaration of Papert: "I do not present LOGO environments as my proposal for this.... [they are] too primitive... too limited by the technology of the 1970s...". The author of this paper aims to answer the question: How do the technological advances of today correspond to Papert's vision? The author pays tribute to Papert by making connections between many of his key ideas and today's technological reality. At the same time, taking into account the complexity and the hybrid nature of the emerging smart environment, he raises the question of whether the educational value of Papert's ideas is sufficient to justify the corresponding technological investments? This challenging question is indeed worthy of study.

A comprehensive philosophical analysis of constructionism is presented in Floridi's work (2011a). The author emphasizes that constructionism means a shift from passive and declarative knowledge to interactive and practical knowledge that comes from providing proofs. He summarizes the perspective and the importance of the constructionist approach thus: "Constructionism is a very common and unproblematic approach in all those sciences that have a poietic attitude towards their subject, which they tend not only to study but also to build... They could teach some important methodological lessons to philosophy..." (Floridi, 2011a: 302).

In general, the majority of researchers and educators definitely recognize the constructionist ideas as a fundamental component of education in contemporary society. Nevertheless, to the best of our knowledge, the role and the place of constructionism in forming children's worldview have not been sufficiently studied. In our paper, we intended to fill this vacuum, by studying the evolution of the constructionist approach in the education of the digital society. The assumption underlying the present work is that the approach developed by Seymour Papert underlies the basic components inherent in the worldview of our current digital society.

The rest of the article is organized as follows. Section 2 defines a specific four-component structure of human worldview; it also introduces four global transformations inherent to the digital society. The next four Sections (3 - 6) correspond to the four components of the worldview. Each of the sections is twofold: in the first part of each section, we review the constructionist ideas of the early IT epoch (harbinger of the digital revolution); in the second part, we demonstrate implementations of these ideas in the digital epoch. Conclusions 
are presented in Section 7.

\section{Worldview and Transformations of Society in the Digital Age}

Digital technologies, which appeared only in the late 1970s, have significantly changed our world. The speed and intensity at which these global changes have occurred is nothing short of amazing. At the end of the 20th century, only one percent of the world's population was connected to the Internet. In 2017, more than $50 \%$ of the world's population is online. For the most part, people interact in social networks, search for information, and buy goods and services on the network. They are equipped with mobile devices that provide them with ubiquitous and unlimited access to a huge amount of knowledge in a wide range of areas of human culture. Their lives, their self-perception, and their perception of the world around them have changed radically. The world has been headed this way since the late 1970s, when the first theoretical foundations of the coming digital age were formulated.

The years that transpired until the emergence of IT in education constitute the period of the technologies' applicational development and social absorption, the importance of which cannot be overestimated. First of all, in practical terms, these are network technologies that provided the information infrastructure for the entire planet, connecting people and giving them almost unlimited access to knowledge of a diverse nature. Furthermore, the secondary developments, such as Web 2.0, mobile applications, the cloud, and the Internet of things, not only changed the world, but led to changes in people's consciousness.

Both the intensive development and the widespread use of IT have had a radical impact on the human condition, with enormous ethical, legal and political consequences. Moreover, informational technologies are gradually and increasingly affecting the most important components of our worldview (Floridi, 2015):

1) Our self-conception (who we are);

2) Our mutual interactions (how we socialize);

3) Our conception of reality (our metaphysics); and

4) Our interactions with and impact on reality (our agency).

These influences are caused by at least four fundamental transformations taking place in our society in the digital age (Ess, 2012).

a) Shifting from the primacy of entities over interactions to the primacy of interactions over entities

Traditionally, people have paid more attention to what entities are, and considered the interactions between them as a secondary phenomenon. In addition, fragmentation is considered a negative characteristic and unity is viewed as superior to fragmentation. Similarly, in framing our relations with others, we often speak of relationships in binary terms, as either flowing or hindered. With the digital transition, the importance of interactions has become central. The pri- 
macy of interactions becomes obvious, while the identity is seen as the result of the interactions.

\section{b) Reversing from scarcity to abundance, when it comes to information}

Since ancient times, people have assumed that if only we knew everything there is to know, we would act perfectly. In other words, "to err is human", because human kind does not possess the ability to grasp all of the knowledge and information in the world. This encyclopedic ideal considers nature to be the only reliable source of knowledge. Following the digital transition, the foundation underlying this ideal has shifted from natural resources to information. As the number of human digital activities has grown exponentially, and all kinds of electronic devices and various social networks leave a recorded trace of peoples' activities. Our traditional aim-encyclopedic knowledge-has changed now that we navigate through the world of the information abundance.

c) Blurring the distinction between reality and virtuality

People are accustomed to dualistic thinking. Our perception of reality and virtuality as separate and clearly distinct remained unquestionable over several centuries. Emerging technologies have made virtuality more real than ever before. As a result, the new digital reality undermines the real/virtual divide. As a result, many other perceived dualities are being questioned and reconsidered. Previous distinctions are being blurred. We live today in a new digital reality, which is comprised not only of "purely real" and "purely virtual" objects, but also of objects of a new, combined nature.

d) Blurring the distinctions between human, machine and nature

In general, people easily distinguish humans from artifacts and nature. Nevertheless, the phenomenon of blurring the distinction has been known since the Darwinian revolution and the industrial era. After Darwin, we acknowledged that we are part of nature, in full continuity with animals. Since the industrial era, artifacts and nature have become intrinsically connected. The digital transition acts as a powerful accelerator of the blurring of these distinctions. Our worldview is still based on the traditional and effective distinctions between humans, nature and artifacts. Nevertheless, it has to be adapted to the new reality, where these distinctions no longer exist (Floridi, 2015).

The intersection and the interrelation of the above two domains-the human worldview and the digital transformations of society-are the focus of our study. In general, each transformation affects all of the components of the human worldview. In our study, we show that in the context of constructionism, behind each of the components that make up our worldview (1)-(4) there is a major, specific digital transformation (a)-(d). In other words, each specific component of our worldview is mainly affected by one type of transformation. We then discuss how these components have changed in the period since the origin of $\mathrm{Pa}$ pert's constructionist approach (late 1970s) until the present digital era.

\section{Activating the Perception of Ourselves}

Let us start with the reflexive component of the worldview-the perception of 
ourselves. Papert's perception of the PC as a means of activating cognition was the starting point of his interest in education. The idea that a computer can be used by the student to create a learning environment that constitutes a complete and flexible universe has become one of the central ideas of constructionism. Learners' ability to be the creators of their own learning environment affects their self-perception. Hence, the well-established traditional concept of self-perception as a certain and stable entity is changing. Creating their own unique microworld stimulates learners to play an active and dynamic role in the learning process. We refer to this phenomenon as the activation (intensification) of the learner's self-perception.

\subsection{Activating Self-Perception in the Early IT Epoch}

For the inhabitant of the industrial society, personalized learning was quite limited. Learners studied the world as a team: in a classroom, in a group, or within the education system. In all of these frameworks, the individual is merely an element in a large system, and it is within this system that one's personality is formed and remains essentially unchanged.

The most famous idea of Papert is that "instructionism", the approach to learning based on instructions that determine what the student should remember, must give way to "constructionism", according to which the individual actively constructs concepts and knowledge using elements of the educational content. Papert makes a fundamentally important addition to Piaget's famous thesis that "every child is the architect of his own intellectual structures", namely, in order to exercise the freedom to construct, the appropriate environment is necessary. In such an environment, the child becomes "the architect of his personal microworld". Papert expresses this idea that the student's activity consists in the creation of his or her own artificial microworld in the very first lines of his book (Papert, 1980). He reminisces about the gears with which he played in his childhood and on the basis of which he created his own microworlds. Building these environments was a process of active learning and, crucially, it was a basis for forming his "self-knowledge".

Called by Papert "a machine that brings back a natural character to learning", the computer becomes a means for learning. Using the computer, students, just like young Seymour with his gears, come to realize that they have the capacity to be the creators- "the architects" - of new environments and, simultaneously, of their new intellectual structures, which constitute the most important component of the learning. Papert emphasized the active, individual, emotional and very personal nature of this type of educational activity that changes the child's self-perception. In this manner, this new self-perception becomes activated.

\subsection{Activation of Self-Perception in the Digital Age}

The network-driven society is characterized by the hyperconnection of its inhabitants, when everything is interconnected: people with people, people with ma- 
chines, and machines with machines. In such conditions, the activation of self-perception is substantially enriched; the personal environment becomes a part of a hyperconnected world, where the self-perception of the individual radically changes. A networked personality is formed and developed not only in the traditional reality, but also in virtual cyberspace, in the infosphere. This new type of personality formation occurs under the conditions of an emerging phenomenon called Personal Identity Online (Floridi, 2011b; Rodogno, 2012).

Obviously, activation of self-perception in the case of the online personality is different from the activation of the habitual offline personality. For Papert, personalization of the environment was associated with physical objects, or with computer simulations-microworlds. In his works and public lectures, he predicted the emergence of mobile personal environments, talking for example, about miniature computers and even wearable technologies. However, 30 - 40 years ago, no one could have imagined miniature devices connected to global networks. In effect, mobile technology has brought the activation of self-perception to a new level.

In today's networked society, a person is permanently connected with his or her personal environment. This connection is provided through personal mobile devices equipped with properties of contextual and social awareness (Lukowicz et al., 2012). Due to these properties, in addition to personal identification details, the personal environment conveys a person's location and cultural context. At the same time, the personal environment, being a part of the global infosphere, connects one with a huge variety of information resources and social networks. Thus, the individual is immersed in a world of numerous interactions, where one's personality is identified and functions. In these conditions, one's self-perception undergoes a change. Now the determining factors of self-perception are the connections and interactions in which one participates. This manifests one of the transformations caused the digital transition mentioned in Section 2, namely, the shift from the primacy of entities over interactions to the primacy of interactions over entities.

\section{Democratization of Interactions with Others}

In the context of education, the second component of our worldview, interactions with others, determines the perception of the student's place in the school: through one's relations with other students and teachers, students recognize their role in the educational process. The approach suggested by Papert is highly democratic, anti-authoritarian and, in this sense, revolutionary. This social aspect of Papert's approach is the one most widely known to the public and it is occasionally the target of public criticism.

\subsection{Democratization of Education in the Early IT Epoch}

Papert notes that using a computer as a learning tool is in conflict with the traditional education system, which is founded on hierarchical principles. This dis- 
tinction was highly significant to him. In his work "Perestroika and Epistemological Politics" (Papert, 1990), he notes that the use of new technologies can destabilize the existing educational system. The epistemological conflict that $\mathrm{Pa}-$ pert discovered is the contradiction between two views of education: on the one hand, education is conceptualized as a hierarchical and centralized system, distanced from the individual student, and on the other hand, it takes the form of a heterarchical, decentralized, and personalized system. He characterizes this contradiction as a confrontation between traditional forms of teacher-centered teaching and approaches that place the students and their intellectual growth at the center of the pedagogical process. To explain his ideas, Papert uses an analogy with the case of Soviet "Perestroika" that was very popular at the time. Indeed, everything that Gorbachev tried to improve in the old Soviet system by reconstructing it through incremental changes, was failing. The system collapsed, and, according to Papert, the same will also happen with the traditional centralized education system, with its unified curricula, textbooks, exams, etc. Papert's idea to replace the old obsolete educational system with a new decentralized one is the democratization of schools. We consider the second component of the constructionist worldview (our mutual interactions and the way we socialize) as initiated by the ideas of democratization of education.

\subsection{Democratization of Education in the Digital Age}

In 1990, democratization of education was a dream-like notion. Today, in the digital age, this idea has a real embodiment both in pedagogical theory and practice. Goldin (2009) formulated three basic principles of the democratization of education: subjecthood, redundancy, and cooperation.

Subjecthood. In the traditional class-lesson system, the content of education is determined by the unified curriculum. In digital-age education, the content of study is the students' activities. Therefore, the curriculum does not exist without the student and, hence, the content of the teaching becomes subjective. In other words, the students themselves form a type of "ad-hoc" curriculum. This is done on the basis of almost unlimited access to a variety of network resources and according the students' own educational trajectories. Any knowledge becomes subjective. It can be considered a generalization of a personal experience, the result of the personal structuring of this experience. The school ceases to be a place for the transmission of knowledge to students, and instead becomes a place intended for the exchange of subjective knowledge between the students and the teacher.

Redundancy is a fundamental phenomenon that is necessary for the implementation of subjectivity. Students' personal knowledge develops within an educational environment, which is saturated with a variety of literatures, consulting opportunities, network resources, forums and more. Such a deliberately excessive educational environment allows students to accumulate the active experience necessary for the development of personal knowledge, to build a personal educational trajectory. The function of the teacher in a digital school is not 
to broadcast the contents of the curriculum, but rather to organize the diverse activities of students in the new educational environment.

Cooperation. The traditional class-lesson system is based on coercion, and the leading motivation for learning in the traditional class is the grade. The digital school is a school of cooperation, where the educational process is based on the equality of participants: the distinction between the concepts of "teacher" and "student" is gradually blurred and, consequently, students and teachers become equal partners in the educational process.

The radical democratization of education in the digital age would be impossible without the above phenomenon of redundancy, i.e., unlimited access to knowledge. This is made possible by the transformation (defined in Section 2) from information scarcity to information abundance, an outcome of the digital transition.

\section{Virtualization-A New Concept of Reality}

The concept of reality is one of the most important components of the Papert's theory. This concept is undergoing fundamental changes in today's digital society. The focus of the new concept of reality is the relationship between the real and virtual worlds, which corresponds to the phenomenon defined in Section 2, namely, the "blurring of the distinction between reality and virtuality".

\subsection{Virtualization in the Early IT Epoch}

Virtualization is a fundamental phenomenon in the context of integrating IT in education. It follows directly from the constructionist approach. When learners create their own specific and unique environments, the result is the simultaneous existence of real microworlds (for example, from the LEGO kit) and virtual (computer simulation based) microworlds, which are mutually complementary. Although virtual microworlds became the main motif and component of $\mathrm{Pa}$ pert's approach, he always stressed the necessity and importance of having students practice using also real physical objects. The "real vs. virtual" dilemma has always been the subject of his reflection and study. Papert realized that this problem has both ontological and epistemological depth, as it has important implications for children's conceptualization of reality, and for their understanding how to study the surrounding world. In the pre-network era of IT, virtualization was manifested in the form of computer microworlds. In those days, the real and virtual worlds coexisted and functioned independently, separately from each other. At that time, there was no reason to talk about the virtualization of reality. Even in the early 1990s, in the most authoritative philosophical publications, the dichotomy "real vs. virtual" was formulated as a fundamental paradigm. In a networked society, the situation has changed significantly.

\subsection{Virtualization in the Digital Age}

Today, we live in a world where the distinctions between reality and virtuality 
are blurred. On the one hand, this is a direct consequence of improving the quality of virtualization, that is, virtual objects become indistinguishable from real ones. On the other hand, there is a symmetric process of the virtualization of habitual real objects. This is the result of the massive integrating of digital and hybrid technologies in the objects that surround us. Increasingly, in the majority of human activities, success depends on the ability to respond adequately and effectively to events-not in face-to face, real situations-but through the ever-present computer interface. Moreover, often the computer interface simply replaces reality, even in trivial situations. For example, whereas once people used an outdoor thermometer to determine the temperature outside, today they are most likely to look for external temperature reports on the Internet or by using a corresponding mobile application.

A clear example of the blurring the distinction between reality and virtuality is the "mixed reality" technology, which is the most promising today in terms of its use in education (Xie, 2012). The name itself speaks about the idea of using both real and virtual objects simultaneously. This type of environment is the natural continuation of Papert's constructionist ideas.

Obviously, the rejection of the traditional dichotomy "reality vs. virtuality" and the perception of a new environment in which the distinctions between the real and the virtual are blurred is an important characteristic of the perception of reality in the digital era.

\section{Integration with the Surrounding Reality}

The concept of integrating with reality is of particular interest, according to Papert. At the heart of this concept is the idea that the student merges with his or her own learning environment.

\subsection{Integration with the Surrounding Reality in the Early IT Epoch}

According to the constructionist approach, in the process of learning, the learning environment becomes an active part of the ongoing process. This can be illustrated by the classic example of teaching children the notion of a circle by using LOGO. To solve the problem of constructing a circle, children are encouraged "to teach" a turtle how to draw a circle. To this end, Papert invites children to imagine themselves as a turtle and execute instructions according to the corresponding LOGO procedure. The turtle was invented as an "object uniting culture, knowledge and the possibility of personal identification". It became an accomplice of the child in games, a partner in studying the surrounding world. It performs the role of a specific "subject", designed to reflect the "second self" of the child. This situation leads to awareness of the learning environment as unique and inseparable from the learner. When the environment is thus integrated in the personal sphere, humans' interaction with reality strongly corresponds to the constructionist approach. Such interaction can be interpreted as the 
beginning of a person's convergence with the surrounding world, the convergence of the subject and the object.

\subsection{Integration with the Surrounding Reality in the Digital Age}

One of the important transformations of the digital era is the phenomenon of the blurring the distinctions between humans, machine and nature (see Section 2). Throughout most of human history, artifacts were easily distinguished from nature. This distinction was lost in the industrial era, when people realized that they are an organic part of nature. Since the industrial era, both artifacts and nature have become an integral part of the industrial development process. Later, thanks to the success of biotechnology and medicine, people and artifacts also became connected (Ess, 2012). Recently, due to the mass integration of various sensors in human life and the progress made in cognitive sciences and biotechnology, the difference between people and artifacts has become even more blurred.

First of all, in the digital age, the surrounding reality is comprised of the "Internet of things", with various "smart" objects interacting through the network. These objects, in addition to their usual physical parameters, have the ability to interact with other objects and change in the process. In addition, artificial objects of this type, unlike natural objects, are open systems, which can be reprogrammed (Wing, 2006). Awareness of the above-mentioned properties makes students' perceptions of objects nowadays significantly different from the prior traditional perception, more aligned with the famous "gears" of Papert's childhood.

Nowadays, students are "permanent residents" of the global network, equipped with mobile and portable technologies. These, along with the emergence of various sensors, turn the student into a "networked learner". The environment itself seems to be an assimilated part of the individual. The interaction is no longer between external objects and the traditional student, but between the mixed-real-and-virtual environment and the informational organism (inforg), assimilated in the environment.

Thus, it becomes impossible to draw a line between personality and the environment. Hence, in the digital age, a new perception of our interaction with the surrounding reality is formed. It is the interaction based on the complete or partial merging with the reality.

Today, the evolution of Papert's initial idea about the "machine that brings back a natural character to learning" is clearly visible. In Papert's childhood, this role was played by gears and similar mechanical objects; in the years of the origin of the constructionism, these were replaced by computers and LOGO; today, in the digital age, the learner and the "machine" are becoming a single entity. The surrounding reality, containing "smart objects" and populated by "networked students", becomes a reality, in which the object and the subject converge. This integration is the main feature of a human's interaction with reality in the digital age. 


\section{Conclusion}

In this article, we studied the evolution of the constructionist learning approach, from the early IT epoch through the digital age. The main contribution of the study is the formulation of a number of trends in the evolution of constructionism, each of which, in turn, corresponds to a certain component of human worldview. We identified the following trends:

- Activation of the perception of self, that is, our self-conception becomes an active, dynamic entity;

- Democratization of our mutual interactions with others, which has replaced the traditional hierarchy;

- Virtualization of our conception of reality;

- Integration of the environment as an intrinsic part of the subject and his or her interactions with reality.

We also showed that the above trends are affected by global transformations of the digital age. Each of these trends is affected by a specific digital transformation, as follows:

- In the activation of self-perception, we have experienced a shift from the primacy of entities over interactions to the primacy of interactions over entities.

- The democratization of interactions with others is accelerated by a shift from scarcity to abundance of information.

- Virtualization of our perception of reality is further advanced by blurring the distinction between reality and virtuality.

- Integration of the subject and the object is characterized by the blurring of the distinctions between human, machine and nature.

In the late 1970s, Seymour Papert noticed the early emergence of these trends; they are embodied in his constructionist approach to education. Taking into account the aforementioned trends and their evolution from the IT era to the digital age, we suggest that Constructionist ideas may be considered a theoretical model of what should constitute education in the digital era.

\section{References}

Beynon, M. (2016). Mindstorms Revisited: Making New Construals of Seymour Papert's Legacy. In International Conference EduRobotics 2016 (pp. 3-19). Cham: Springer.

Blikstein, P. (2013). Gears of Our Childhood: Constructionist Toolkits, Robotics, and Physical Computing, Past and Future. In Proceedings of the 12th International Conference on Interaction Design and Children (pp. 173-182). New York: ACM.

Ess, C. (2012). At the Intersections between Internet Studies and Philosophy: "Who Am I Online?" Philosophy \& Technology, 25, 275-284.

Feurzeig, W. (2010). Toward a Culture of Creativity: A Personal Perspective on Logo's Early Years and Ongoing Potential. International Journal of Computers for Mathematical Learning, 15, 257-265.

Floridi, L. (2011a). A Defence of Constructionism: Philosophy as Conceptual Engineering. Metaphilosophy, 42, 282-304. 
Floridi, L. (2011b). The Construction of Personal Identities Online. Introduction. Minds and Machines (pp. 1-3). Berlin: Springer.

Floridi, L. (2014). The Fourth Revolution: How the Infosphere Is Reshaping Human Reality. Oxford: OUP.

Floridi, L. (2015). The Onlife Manifesto. Berlin: Springer-Verlag GmbH.

Freud, S. (1955). A Difficulty in the Path of Psycho-Analysis. In the Standard Edition of the Complete Psychological Works of Sigmund Freud, (1917-1919): "An Infantile Neurosis" and Other Works (Vol. XVII, pp. 135-144). London: The Hogarth Press and The Institute of Psycho-Analysis.

Goldin, A. M. (2009). Education 2.0. A Teacher's View. Computerra Online: Electronic Journal. http://old.computerra.ru/readitorial/393364/ (in Russian)

Lukowicz, P., Pentland, S., \& Ferscha, A. (2012). From Context Awareness to Socially Aware Computing. IEEE Pervasive Computing, 11, 32-41.

Papert, S. (1980). Mindstorms: Children, Computers, and Powerful Ideas. New York: Basic Books, Inc.

Papert, S. (1990). Perestroika and Epistemological Politics. Cambridge, Massachusetts: Epistemology and Learning Group, MIT Media Laboratory.

Resnick, M. (2012). Reviving Papert's dream. Educational Technology: The Magazine for Managers of Change in Education, 52, 42-46.

Rieber, L. P. (2004). Microworlds. Handbook of Research for Educational Communications and Technology, 2, 583-603.

Rodogno, R. (2012). Personal Identity Online. Philosophy \& Technology, 25, 309-328.

Wing, J. M. (2006). Computational Thinking. Communications of the ACM, 49, 33-35.

Xie, C. (2012). Framing Mixed-Reality Labs. Concord Consortium. Spring: Newsletter, 2012. https://concord.org/newsletter/2012-spring/framing-mixed-reality-labs/ 